\title{
Echinophora platyloba DC (Apiaceae) Crude Extract Induces Apoptosis in Human Prostate Adenocarcinoma Cells (PC 3)
}

\author{
Fatemeh Zare Shahneh ${ }^{1,2}$, Behzad Baradaran ${ }^{1,2}$, Jafar Majidi $^{1,2}$, Zohreh Babaloo $^{2}$
}

Background: Prostate cancer is the second leading malignancy worldwide and the second prominent cause of cancer-related deaths among men. Therefore, there is a serious necessity for finding advanced alternative therapeutic measures against this lethal malignancy. In this article, we report the cytotoxicity and the mechanism of cell death of the methanolic extract prepared from Echinophora platyloba DC plant against human prostate adenocarcinoma PC 3 cell line and Human Umbilical Vein Endothelial Cells HUVEC cell line.

Methods: $\quad$ Cytotoxicity and viability of the methanolic extract were assessed by 3-(4,5-dimethylthiazol-2-yl)-2,5-diphenyltetrazolium bromide (MTT) assay and dye exclusion assay. Cell death enzyme-linked immunosorbent assay (ELISA) was employed to quantify the nucleosome production resulting from nuclear DNA fragmentation during apoptosis and determine whether the mechanism involves induction of apoptosis or necrosis. The cell death was identified as apoptosis using terminal deoxynucleotidyl transferase (TdT)-mediated dUTP nick end labeling (TUNEL) assay and DNA fragmentation gel electrophoresis.

Results: $\quad$ E. platyloba could decrease cell viability in malignant cells in a dose- and time-dependent manner. The IC50 values against PC 3 were determined as $236.136 \pm 12.4,143.400 \pm 7.2$, and $69.383 \pm 1.29 \mu \mathrm{g} / \mathrm{ml}$ after 24,36 , and $48 \mathrm{~h}$, respectively, but there was no significant activity in HUVEC normal cell (IC50 > $800 \mu \mathrm{g} / \mathrm{ml}$ ). Morphological characterizations and DNA laddering assay showed that the methanolic extract treated cells displayed marked apoptotic characteristics such as nuclear fragmentation, appearance of apoptotic bodies, and DNA laddering fragment. Increase in an early apoptotic population was observed in a dose-dependent manner. PC 3 cell death elicited by the extract was found to be apoptotic in nature based a clear indication of TUNEL assay and gel electrophoresis DNA fragmentation, which is a hallmark of apoptosis.

Conclusions: In summary, the E. platyloba extract attenuated the human prostate adenocarcinoma cell proliferation in vitro possibly by inducing apoptosis. E. platyloba is likely to be valuable for the treatment of human prostate adenocarcinoma.

(Biomed J 2014;37:298-304)

Keywords: anti-cancer, apoptosis, cytotoxic, Echinophora platyloba DC, human prostate adenocarcinoma

From the ${ }^{1}$ Immunology Research Center, Tabriz University of Medical Science, Tabriz, Iran; ${ }^{2}$ Department of Immunology, Faculty of Medicine, Tabriz University of Medical Science, Tabriz, Iran

Received: Jun. 22, 2013; Accepted: Nov. 20, 2013

Correspondence to: Prof. Behzad Baradaran, Immunology Research Center, Faculty of Medicine, Tabriz University of Medical Sciences, Tabriz, Iran. Daneshgah St., 51664 Tabriz, Iran. Tel: 98-411-3364665; Fax: 98-411- 3364665; E-mail: behzad_im@yahoo.com

DOI: $10.4103 / 2319-4170.125653$ 
$\mathrm{P}$ rostate cancer is the second leading worldwide malignancy and the second prominent cause of cancer-related deaths among men. ${ }^{[1]}$ According to Facts 2013, it is estimated that there would be 241,740 new cases of prostate cancer diagnosed in the United States in 2013, and prostate cancer is the most frequently diagnosed cancer in men, besides skin cancer, with an estimated 28,170 deaths in 2013. ${ }^{[2]}$

A combination of the treatment options (radiation therapy, brachytherapy, cryosurgery, chemotherapy, hormonal therapy, and surgery) is often recommended for managing prostate cancer. Among the conventional modalities for prostate cancer treatment, chemotherapeutic drugs lead to various side effects in patients. ${ }^{[3]}$ Nowadays, natural anticancer drugs derived from medicinal plants that selectively induce apoptosis and growth arrest in cancer cells without cytotoxic activity in normal cells are available and these natural products could serve as chemotherapeutic agents. ${ }^{[4]}$

Many plant-derived chemotherapeutic agents such as paclitaxel (from Taxus brevifolia), camptothecin (from Camptotheca acuminata), podophyllotoxin (from Podophyllum emody), and vinblastine (from Catharanthus roseus) have been proven as potential sources of anticancer drugs. ${ }^{[5]}$

Apoptosis as a regulated cellular process is induced by many chemotherapeutic agents in cancer treatment. Apoptosis is characterized by particular morphological changes, including plasma membrane blebbing, cell shrinkage, chromatin condensation, and DNA fragmentation. ${ }^{[6]}$ The induction of apoptosis in tumor cells is considered valuable in the management and therapy as well as in the prevention of cancer. The insufficient balance between cell proliferation and apoptotic death leads to cancer, contributing to spread and tumor progression. ${ }^{[7]}$

The genus Echinophora from Apiaceae (Umbelliferae) family, Apioideae subfamily, Echinophoreae tribe is widespread in high latitudes (1400-2000 m above sea level) of northwest of Iran, with four species: Echinophora cinerea, Echinophora platyloba, Echinophora orientalis, and Echinophora sibthorpiana. ${ }^{[8]}$ The essential oil composition of air-dried aerial parts of E. platyloba was analyzed by gas chromatography/mass spectrometry (GC/MS) and was found to contain monoterpene-rich, sesquiterpenes. Phytochemical studies on E. platyloba revealed the presence of betulinic acid, oleanolic acid, and ursolic acid. Preclinical studies have indicated that the betulinic acid and ursolic acid isolated from various plants show cytotoxic and anti-tumor activity. Betulinic acid and ursolic acid are known to exert apoptosis-inducing activity against different malignant cells..$^{[9]}$

For therapeutic purposes, this plant has been applied for its antiseptic, antimicrobial, antifungal, antispasmodic, and anti-tumor activities. E. platyloba is widely used in western and central Iran as a food seasoning and edible vegetable. ${ }^{[10]}$
Previous reports revealed that E. platyloba extract inhibited the growth of mouse fibrosarcoma cell line (WEHI-164) in a dose- and time-dependent manner and inhibited the cell proliferation and induced apoptosis. ${ }^{[11]}$ In this study, the apoptosis-inducing effects of E. platyloba extract were investigated in Human Prostate Adenocarcinoma PC 3 cell line and Human Umbilical Vein Endothelial Cells HUVEC cell line in vitro. Cell cytotoxicity and viability was examined by the 3-(4,5-dimethylthiazol-2-yl)-2,5-diphenyltetrazolium bromide (MTT) and dye exclusion assay. IC50 (concentration that inhibited cell proliferation by $50 \%$ ) was calculated from the dose-response curves by regression analysis. The apoptosis was determined by cell death detection and specific DNA fragmentation with cell death detection by [enzyme-linked immunosorbent assay (ELISA)], terminal deoxynucleotidyl transferase (TdT)-mediated dUTP nick end labeling (TUNEL) assay, and DNA fragmentation gel electrophoresis.

\section{METHODS}

\section{Preparation of plant extract}

E. platyloba plants were collected from the Shahre Kord (west of Iran) during June 2011. E. platyloba is a perennial plant, distributed only in the Mediterranean region and some central and western provinces of Iran. The Department of Botany, Institute of Medicinal Plants (IMP) of Karaj, Iran identified the plant. A voucher specimen was deposited in the herbarium of the above-mentioned department (217 IPM).

The aerial parts of the plant were separated, air-dried, and finely powdered. Extraction was carried out by macerating $100 \mathrm{~g}$ of powdered dry plant in $500 \mathrm{ml}$ of $70 \%$ methanol for $48 \mathrm{~h}$ at room temperature. Then, the macerated plant material was extracted with $70 \%$ methanol solvent by percolator apparatus (2-1 volume) at room temperature. The crude natural product extract was removed from the percolator, filtered through Whatman filter paper (no. 4), and dried under reduced pressure at $37 \mathrm{C}$ with rotator evaporator before being added to methanol as the solvent. The methanol extract was filtered and concentrated using a rotary evaporator and then evaporated to dryness. Twenty milligrams of the dried extract was dissolved in $100 \mu$ l dimethyl sulfoxide (DMSO) and diluted with $3.90 \mathrm{ml}$ RPMI-1640 to give a concentration of $5000 \mu \mathrm{g} / \mathrm{ml}$.

\section{Cell culture}

The PC 3 cell line of human prostate adenocarcinoma cells (NCBI code C427) and HUVEC cells (NCBI code C554) were obtained from Pasteur Institute of Iran (cell bank). Both cell lines were cultured in RPMI-1640 (Sigma-Aldrich, Germany $\mathrm{pH}=7.2$ ) containing $10 \%$ fetal calf serum (FCS) and antibiotics $(100 \mathrm{U} / \mathrm{ml}$ penicillin, 
$100 \mu \mathrm{g} / \mathrm{ml}$ streptomycin), and placed at $37 \mathrm{C}$ and $5 \% \mathrm{CO}_{2}$ in an incubator overnight. Ten thousand cells from the log phase cultures were seeded in $100 \mu \mathrm{l}$ of RPMI medium supplemented with $10 \%$ FCS per well of 96-well flat-bottom culture plates (Nunc, Roskilde, Denmark). The cells were incubated with different concentrations of E. platyloba extract for a defined time interval $(24,36$, and $48 \mathrm{~h}) .^{[12]}$

\section{Cytotoxicity assay}

MTT assay is one of the most useful tests for investigating cell viability and the cytotoxic effects of drugs, cosmetics, and food additives. Briefly, $1 \times 10^{4}$ viable cells/well were seeded into the 96-well tissue culture plates (Nunc, Denmark), and then, incubated at $37 \mathrm{C}$ overnight in a triplicate manner. Next day, when the cells reached $>80 \%$ confluence, the media were replaced with $200 \mathrm{ml}$ of fresh complete medium containing 50, 100, 200, 300, 400, 500, 600 , and $800 \mu \mathrm{g} / \mathrm{ml}$ concentrations of the crude extract. No plant extract was added to negative controls, but the same amount of DMSO was added to eliminate its intervening effects, and taxol was used as the positive control. Taxol, which contains paclitaxel as the main active compound, is used in chemotherapy of cancer. At different time periods (24, 36 , and $48 \mathrm{~h}$ ), the supernatants were discarded and cell layers were washed with phosphate-buffered saline (PBS, Invitrogen Gibco, Carlsbad, CA, USA) and incubated with MTT $(50 \mu l, 2 \mathrm{mg} / \mathrm{ml})$ in RPMI-1640 without FCS for $4 \mathrm{~h}$ in a humidified atmosphere at $37 \mathrm{C}$. Subsequently, $200 \mu \mathrm{l}$ of DMSO (Sigma) and $25 \mu 1$ Sorensen's glycine buffer $(0.1 \mathrm{M}$ glycine, $0.1 \mathrm{M} \mathrm{NaCl} ; \mathrm{pH}=10.5$ ) were added to dissolve the formazan crystals formed. The optical density (OD) of the colored solution was quantified at $570 \mathrm{~nm}$ wavelength by an ELISA Reader (Bio-Rad, Hercules, CA, USA). ${ }^{[13]}$ The absorbance of untreated cells was considered as $100 \%$. Each extract and control was assayed in triplicate in three independent experiments. The cytotoxic effects of the extracts were estimated in terms of growth inhibition percentage and expressed as IC50, which is the concentration of compound that reduces the absorbance of treated cells by $50 \%$ with reference to the control (untreated cells). It was determined that IC50 values with cytotoxicity results were more than $50 \%$ at screening concentrations. Percent growth inhibition of cells exposed to treatments was calculated as follows: $\%$ inhibition $=100($ treated OD/non-treated OD $) \times 100)$.

\section{Trypan blue assay}

Cell viability was evaluated by trypan blue exclusion assay. Briefly, $1 \times 10^{4}$ cells/well were seeded into 96-well culture plates and treated with or without $[0.1 \%(\mathrm{v} / \mathrm{v}) \mathrm{DMSO}]$ crude extract at specified doses for 24,48 , and $36 \mathrm{~h}$. After the incubation period, the cultures were harvested and washed twice with PBS. The cell pellet was then resuspended with
$0.5 \mathrm{ml}$ PBS. Then, $20 \mu \mathrm{l}$ of cells was mixed with an equal volume of $0.5 \%$ trypan blue (Merck, Darmstadt, Germany), and the plates were incubated for $2 \mathrm{~min}$. The number of viable and non-viable cells was counted using Neubauer hemocytometer (Weber, Teddington, England) by clear field microscopy (Nikon, Tokyo, Japan). ${ }^{[14]}$

The number of viable cells was calculated according to the following formula: [The unstained cell count (viable cells) $\times$ the dilution of the cell suspension $\left.\times 10^{4}\right] /$ the number of hemocytometer squares counted. The percent viability was calculated as: (Viable cells/the total cell count) $\times 100$. Each extract and control was assayed two times in triplicate. The cells were stained with trypan blue and live cells were enumerated. Cell counts were expressed as mean \pm standard deviation (SD).

\section{Cell death detection}

Cell Death Detection ELISA ${ }^{\text {PLus }}$ Kit (Roche, Basel, Switzerland) was used to quantify the histone-complexed DNA fragments (nucleosomes) in the cytoplasm of the apoptotic cells after the induction of apoptosis. Briefly, after incubation with the methanolic extract (at concentrations determined by MTT assay), the 24-h supernatants were collected and cells were pelleted and lysed. Mouse monoclonal antibodies against single-stranded DNA and histones (H1, H2a, $\mathrm{H} 2 \mathrm{~b}, \mathrm{H} 3$, and $\mathrm{H} 4$ ) were specifically detected, and the bound mononuclesomes and oligonucleosomes were derived from the cells undergoing apoptosis. The antibody-nucleosome complexes were fixed to the streptavidin-coated microtiter plate by biotinylated anti-histone antibodies. The anti-DNA antibodies were conjugated with a peroxidase that reacted with the substrate 2,2'-azino-di-(3-ethylbenzthiazoline sulfonic acid) (ABTS) to form a colored product. The remaining steps were carried out according to the instructions supplied by the manufacturer. The resulting color developed, which was proportional to the amount of nucleosomes captured in the antibody sandwich, was measured at $405 \mathrm{~nm}$ wavelength using a Benchmark microtiter plate reader (Bio-Rad). ${ }^{[15]}$ Results were expressed as the apoptotic index, calculated from the ratio of absorbance of the treated (apoptotic) sample to that of the untreated (control) sample.

\section{TUNEL assay}

To assess cell death by apoptosis, an In Situ Cell Death Detection Kit, POD (Roche, Germany) for DNA chromatin morphologic features was used for quantification. The manufacture's guidelines were followed for the procedure. Briefly, PC $3\left(1.5 \times 10^{5}\right)$ cells were subcultured into 6-well plates and incubated for $24 \mathrm{~h}$ at $37 \mathrm{C}$ and $5 \% \mathrm{CO}_{2}$. The cells were treated with extracts at the concentrations required for $50 \%$ inhibition of growth of PC 3 cells (LC50) for $24 \mathrm{~h}$. Negative control cells were treated with the same final concentration 
of DMSO present in the treated wells $[0.2 \%(\mathrm{v} / \mathrm{v})]$. After treatment, the cells were fixed with $4 \%$ (w/v) paraformaldehyde in PBS ( $\mathrm{pH}$ 7.4) for $1 \mathrm{~h}$ at room temperature and rinsed twice with PBS. Then, the fixed cells were incubated with blocking solution ( $3 \% \mathrm{H} 2 \mathrm{O} 2$ in methanol) for $10 \mathrm{~min}$ and rinsed with PBS. The cells were then incubated in permeabilization solution $(0.1 \%$ Triton X-100 in $0.1 \%$ sodium citrate) for $2 \mathrm{~min}$ on ice.

Subsequently, $50 \mu \mathrm{l}$ of reaction mixture containing TdT enzyme and nucleotide was added to the cells and incubated for $1 \mathrm{~h}$ at $37 \mathrm{C}$. After washing three times with PBS, the slides were incubated with $50 \mu \mathrm{l}$ converter-POD, streptavidin HRP solution, for $30 \mathrm{~min}$, and rinsed three times with PBS. Finally, the cells were incubated with DAB (3, 3'- diaminobenzidine) and the stained cells were analyzed with a light microscope. ${ }^{[16]}$

\section{DNA fragmentation assay}

DNA fragmentation was used to investigate the mode of cell death induced by the E. platyloba extract. Briefly, after treatments, 1 million PC 3 cells were treated with IC50 dose of E. platyloba and incubated for $24 \mathrm{~h}$ at $37 \mathrm{C}$ in $5 \%$ $\mathrm{CO}_{2}$ atmosphere. After incubation, the cells were harvested and lysed with $0.1 \mathrm{ml}$ lysis buffer containing 0.5 M EDTA, $0.5 \%$ Triton $\mathrm{X}-100$, and $1 \mathrm{M}$ Tris- $\mathrm{HCl}(\mathrm{pH} 8.0)$, and distilled water, and then centrifuged at $10,000 \mathrm{~g}$ for $30 \mathrm{~min}$. The supernatant was digested with $0.1 \mathrm{mg} / \mathrm{ml}$ proteinase $\mathrm{K}$ at $50 \mathrm{C}$ for $60 \mathrm{~min}$, extracted with phenol/chloroform mixture, and precipitated with two volumes of ice-cold isopropanol. The DNA pellet was then dissolved in appropriate volume of RNase solution $\left(10 \mathrm{mg} / \mathrm{ml}\right.$ RNase I) and incubated at $37^{\circ} \mathrm{C}$ for $30 \mathrm{~min}$. DNA samples were separated by electrophoresis in $1.2 \%$ agarose gels. DNA was stained with ethidium bromide and photographed under UV light transilluminator. ${ }^{[17]}$

\section{Statistical analysis}

Data are expressed as mean \pm SD for at least three independent determinations in triplicate for each experimental point. The data were analyzed using IBM SPSS Statistics 20 software. For all the measurements, two-way analysis of variance (ANOVA) followed by Duncan's New Multiple Range Test $(P \leq 0.05)$ was used to assess statistically significant difference between control and E. platyloba treated cells.

\section{RESULTS}

MTT assay is based on the ability of viable cells with active mitochondria to produce succinate dehydrogenate enzyme which cleaves the tetrazolium rings of MTT, where the OD obtained is proportional to the number of healthy viable cells. In this study, E. platyloba extract showed a time- and dose-dependent inhibition of the PC 3 cell growth. The graph plotted shows the concentrations of the E. platyloba extract that caused 50\% loss of cell viability (IC50) against PC 3 cells as $236.136 \pm 12.4,143.400 \pm 7.2$, and $69.383 \pm 1.29 \mu \mathrm{g} / \mathrm{ml}$ after 24,36 , and $48 \mathrm{~h}$, respectively [Figure 1A]. But in the case of HUVEC, there was no significant activity (IC50 > $800 \mu \mathrm{g} / \mathrm{ml}$ ) [Figure 1B], which means that the extract is less toxic to the normal cells than to $\mathrm{PC} 3$ cells.

PC 3 cells were compared to elucidate the cytotoxicity of both E. platyloba extract and toxol (chemotherapeutic agent, control positive), and showed growth suppression of more than $80 \%$ in $800 \mu \mathrm{g} / \mathrm{ml}$ and $93 \%$ in $20 \mu \mathrm{g} / \mathrm{ml}$ in $24 \mathrm{~h}$ [Table 1]. The viability of PC 3 cells exposed to E. platyloba extract was evaluated by dye exclusion assay in $24 \mathrm{~h}$. Figure 2 shows that the viability of cells exposed to E. platyloba extract at concentrations of 50 and $800 \mu \mathrm{g} / \mathrm{ml}$ decreased from $74.3 \pm 7.8 \%$ and $14.66 \pm 2.5 \%$, respectively.

As determined by MTT assay, the extract at 100, 200, and $300 \mu \mathrm{g} / \mathrm{ml}$ was chosen for each cell line in cell death detection ELISA. Apoptotic cell death is not likely to be the only mechanism involved in the down-regulation of cell growth by E. platyloba. Necrosis remained at a low level $(<10 \%)$ over the entire dose range. The possible mechanism was via induction of apoptosis, as evidenced by the significant increase in nucleosome production at 100, 200, and $300 \mu \mathrm{g} / \mathrm{ml}$ of E. platyloba extract after incubation for $24 \mathrm{~h}$, but the percentage of apoptosis was constant $(42.66 \%$, $49.33 \%$, and $54 \%$, respectively).

Figure 3 shows the morphological changes to PC 3 cells after $E$. platyloba extract treatment for $24 \mathrm{~h}$. Phase-contrast inverted microscope revealed that E. platyloba extract induced cell shrinkage, apoptotic vacuoles, membrane blebbing, and formation of spherical and floating cells in a dose- and time-dependent manner. These changes are

Table 1: Comparison of cytotoxicity activity of (A) Toxol and (B) E. platyloba in $24 \mathrm{~h}$. Data presented are the mean \pm SEM of three independent experiments

\begin{tabular}{lc}
\hline Concentration $(\mu \mathrm{g} / \mathrm{ml})$ & Growth inhibition \% \\
\hline (A) Toxol & \\
20 & 94.78 \\
15 & 86.86 \\
10 & 73.69 \\
5 & 59.88 \\
0 & 9.3 \\
(B) Echinophora Platyloba DC & \\
800 & 86.33 \\
600 & 74.44 \\
500 & 69.66 \\
400 & 64.66 \\
0 & 9.8 \\
\hline
\end{tabular}

Abbreviation: SEM: Standard error of mean 


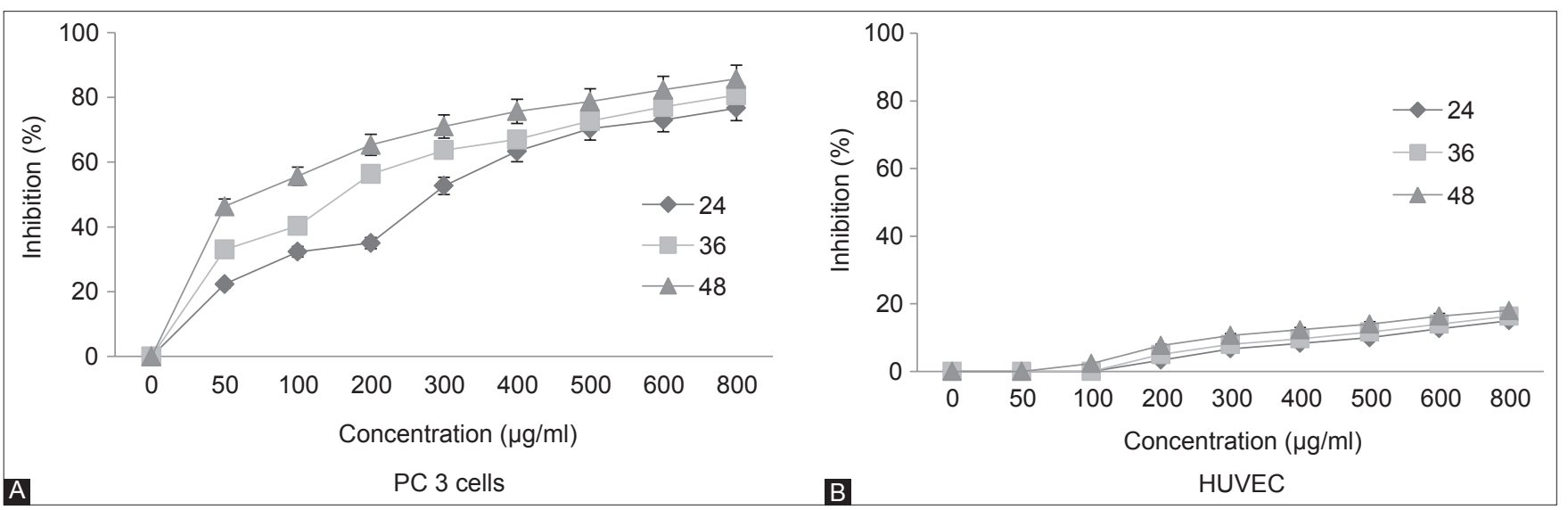

Figure 1: The effects of E. platyloba on proliferation of (A) PC 3 cells and (B) HUVEC. Cells were incubated with increasing concentrations of E. platyloba in culture medium for 24,36 , and $48 \mathrm{~h}$. Data are presented as the mean \pm SEM of three independent experiments. $p<0.05$.

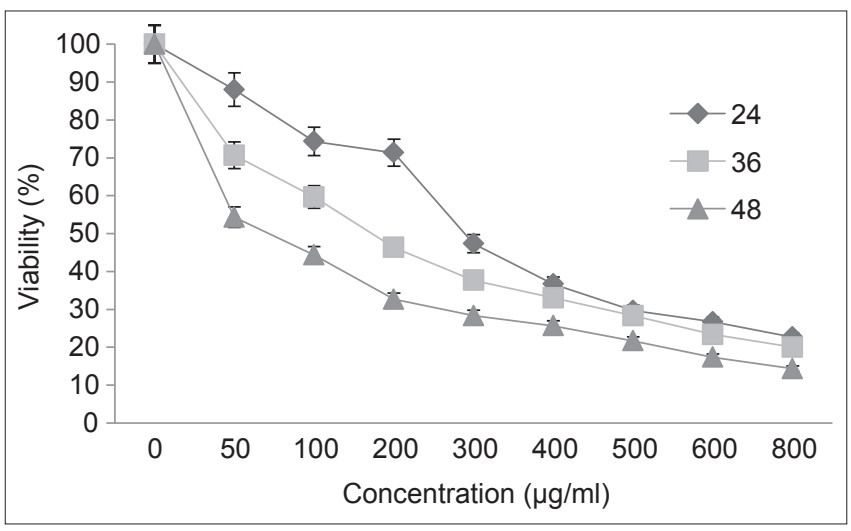

Figure 2: The effects of E. platyloba on the viability of PC 3 cells. Cells were incubated with increasing concentrations of E. platyloba in culture medium for 24,36 , and $48 \mathrm{~h}$. Data are presented as the mean \pm SEM of three independent experiments. $p<0.01$.
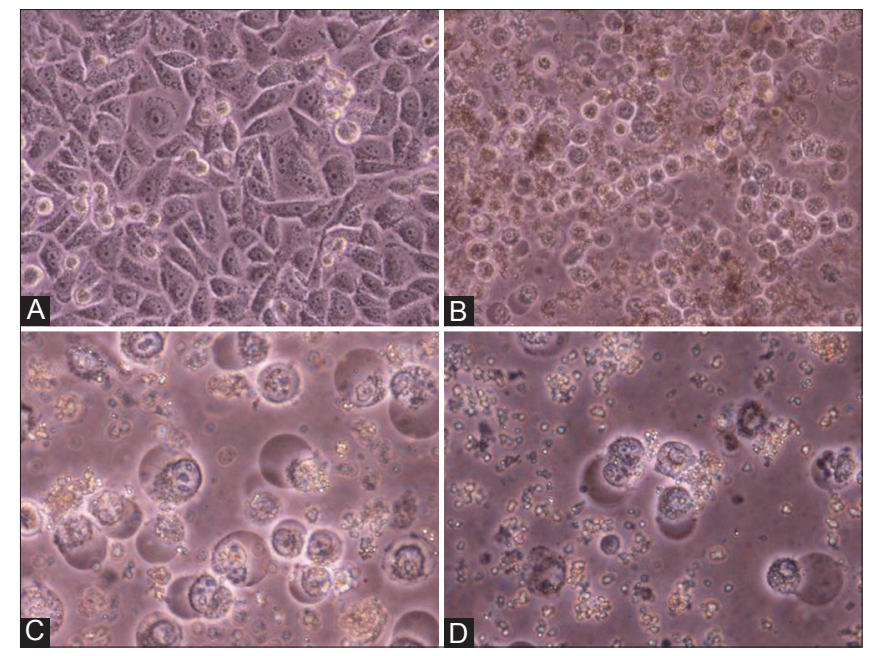

Figure 3: Morphological changes of PC 3 cells being observed under phase-contrast inverted microscope: (A) untreated PC 3 cells and (B-D) cells exposed to 100, 200, and $300 \mu \mathrm{g} / \mathrm{ml}$ E. platyloba for $24 \mathrm{~h}$. Photomicrographs were taken directly from the culture plates with a phase-contrast microscope $(\times 40$ magnification). suggestive of apoptosis. Cells were treated with DMSO only (vehicle) or with media containing 100,200 , and $300 \mu \mathrm{g} / \mathrm{ml}$ E. platyloba extract for $24 \mathrm{~h}$.

TUNEL assay is used to assay the endonuclease cleavage products by enzymatically end-labeling the DNA strand breaks. Terminal transferase was used to add labeled UTP to the $3^{\prime}$ end of the DNA fragments. As shown in Figure 4, numerous TUNEL-positive cells were observed when PC 3 cells were treated with E. platyloba with DMSO only (vehicle) or with media containing $200 \mu \mathrm{g} / \mathrm{ml}$ E. platyloba extract for $24 \mathrm{~h}$, indicating apoptotic cell death of PC 3 prostate cells.

Typical DNA ladder was observed on DNA electrophoresis gel for cells treated with 100,200 , and $300 \mu \mathrm{g} / \mathrm{ml}$ E. platyloba extract for $24 \mathrm{~h}$ [Figure 4]. DNA fragmentation is a hallmark of apoptosis and the detection DNA ladder in agarose gel electrophoresis is commonly used as a biochemical marker for the measurement of apoptosis. Therefore, DNA gel electrophoresis was used to determine the presence of internucleosomal DNA cleavage. The data depicted in Figure 5 illustrate that DNA fragmentations were clearly detectable in PC 3 cells at all treated doses of the extract for $24 \mathrm{~h}$.

\section{DISCUSSION}

A successful anticancer drug should kill or damage cancer cells without causing severe damage to normal cells, which means with minimum side effects. Apoptosis (programmed cell death), characterized by several morphological and biochemical chances, is a key form of cell death occurring in response of cytotoxic treatment with chemotherapeutic agents. ${ }^{[18]}$ Recent studies have revealed that dysregulation of apoptosis process is involved in different malignancies. The induction of apoptosis in tumor cells has been the most common mechanism of anticancer drugs in many cancer therapies. ${ }^{[19]}$ 
Previous studies have revealed that E. platyloba extract possesses cytotoxic and anti-tumor effects. E. platyloba was found to time- and dose-dependently inhibit the proliferation of WEHI-164, mouse fibrosarcoma cell line, without significant effect on mouse nonmalignant cell line (L929). ${ }^{[1]}$ These results are consistent with our present finding that E. platyloba methanolic extract selectively inhibited the tumor cell growth in a time- and dose-dependent manner. The IC50 of the E. platyloba extract on WEHI-164 proliferation was $196.673 \pm 12.4,122.977 \pm 51.7$, and $58.664 \pm 15.2 \mu \mathrm{g} / \mathrm{ml}$, while against PC 3 it was found to be higher, i.e. $236.136 \pm 12.4,143.400 \pm 7.2$, and $69.383 \pm 1.29 \mu \mathrm{g} / \mathrm{ml}$ after 24,36 , and $48 \mathrm{~h}$, respectively.

In the present study, we found that E. platyloba methanolic extract displayed a significant inhibitory effect on the proliferation of prostate cancer cell lines in a dose- and time-dependent manner. Experimental results show that E. platyloba extract is cytotoxic against PC 3 cells, but is less sensitive to HUVEC, based on the high IC50 value. The E. platyloba extract treatment inhibited PC 3 cell growth in a dose- and time-dependent manner [Figure 1A]. Based on the morphological changes identified by phase-contrast inverse microscopy, typical morphological characteristics of apoptosis, such as cell shrinkage, apoptotic vacuoles, and forming majority of the floating cells, were observed [Figure 3]. The viability rate of cells exposed to E. platyloba extract at concentrations of 50 and $800 \mu \mathrm{g} / \mathrm{ml}$ in $24 \mathrm{~h}$ decreased significantly [Figure 2]. However, HUVEC cells were much less susceptible to the cytotoxic effect of E. platyloba extract. Reduction of cell growth can demonstrate either a decreased proliferation rate or enhanced cell death by apoptosis mechanism.

Cell death detection ELISA determined that apoptotic cell death is the dominant mechanism involved in the down-regulation of cell growth by E. platyloba, and necrosis remained at a low level $(<10 \%)$ over the entire dose range. To determine whether the growth inhibitory activity of E. platyloba was related to the induction of apoptosis, the morphological changes of PC 3 cells were

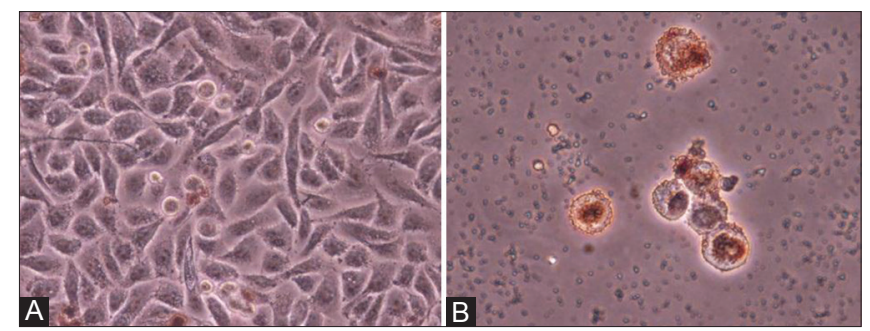

Figure 4: PC 3 cells were treated with (B) $200 \mu \mathrm{g} / \mathrm{ml}$ E. platyloba for $24 \mathrm{~h}$ and (A) DMSO (negative control). Dark stained nuclei PC 3 cells were observed after cell treatment with the extract, whereas no stained nucleus was detected in cells treated with DMSO. Photomicrographs were taken directly from the culture plates with a phase-contrast microscope. $(\mathrm{A}$ and $\mathrm{B}) \times 40$ magnification. investigated under phase-contrast inverted microscope. The morphological changes included condensation of nuclear chromatin, cytoplasmic shrinkage, floating in the medium, formation of apoptotic bodies, blebbing of the cell membrane, and detachment from each other. All these morphological characteristics are the biochemical hallmarks of apoptosis, indicating that apoptosis may play a crucial role in cell death elicited by the E. platyloba on PC 3 cells.

Treatment with the plant extract resulted in degradation of chromosomal DNA into smaller fragments [Figure 5]. Here also, induction of apoptosis, and not necrosis, by the plant extract was confirmed because electrophoresis of DNA of necrotic cells resulted in a smear and not a ladder. Moreover, characteristics of cells undergoing apoptosis were further confirmed with TUNEL assay. Typical morphological features of apoptotic cells in the TUNEL assay were dark brown-stained nuclei and condensed and fragmented nuclei in cells treated with E. platyloba, compared with homogenous nuclear chromatin in the negative control cells [Figure 4]. All these results suggest that E. platyloba extract treatment can induce death of PC 3 cells via apoptosis. However, further detailed investigations of this mechanism are warranted to obtain definite conclusions. The promotion of the specific apoptosis pathway, the key molecular targets that specifically initiate apoptosis of other cancer cells, and the quality standards of $E$. platyloba should be further explored. Thus, E. platyloba would be worth investigating as a novel therapeutic agent originating from a natural source.

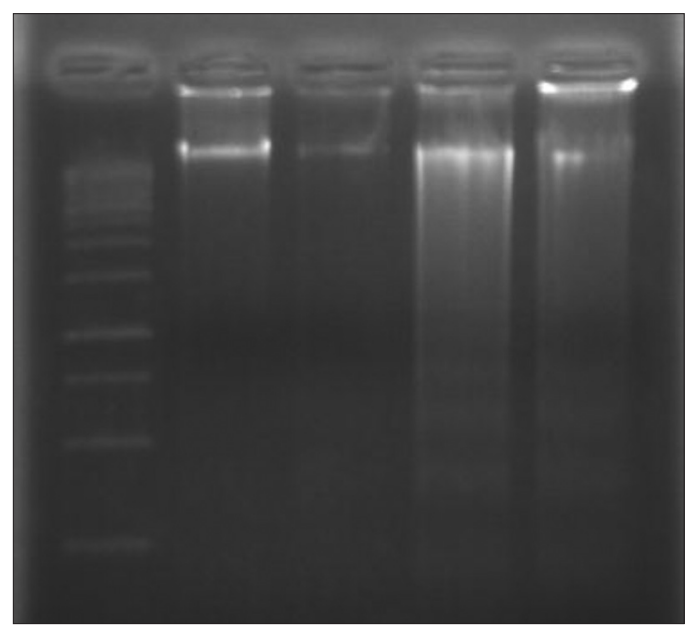

Figure 5: Analysis of genomic DNA fragmentation in PC 3 cells. Cells were treated for $24 \mathrm{~h}$ with crude methanolic E. platyloba extract $(0$, $100,200$, and $300 \mu \mathrm{g} / \mathrm{ml})$. Treatment of PC 3 cells with E. platyloba extract shows DNA ladders by DNA gel electrophoresis. Lane 1: Molecular marker $100 \mathrm{bp}$; lane 2: Untreated cells; lane 3: Treated cells with crude methanolic extract $100 \mu \mathrm{g} / \mathrm{ml}$; lane 4: Treated cells with crude methanolic extract $200 \mu \mathrm{g} / \mathrm{ml}$; lane 5: Treated cells with crude methanolic extract $300 \mu \mathrm{g} / \mathrm{ml}$. 


\section{Conclusions}

This study demonstrates that $E$. platyloba DC extract significantly inhibited PC 3 cell proliferation dose-dependently, especially with IC50 value for cancerous cells lower than that of normal cells, while this extract affected the sensitivity of HUVEC to a lesser extent, possibly via an apoptosis-dependent pathway. These results demonstrate that E. platyloba DC might be a novel and attractive therapeutic candidate for tumor treatment in clinical practice.

\section{Acknowledgments}

This study was supported by the Immunology Research Center of Tabriz University of Medical Sciences and kind assistance of who contributed for this research. Ethical approval was not required.

\section{REFERENCES}

1. Mazhar D, Waxman J. Prostate cancer. Postgrad Med J 2002;78:590-5.

2. American Cancer Society. Cancer Facts and Figures 2013. Vol. 61. Atlanta: American Cancer Society 2013. p. 1-60.

3. Kamesaki H. Mechanisms involved in chemotherapy-induced apoptosis and their implications in cancer chemotherapy. Int J Hematol 1998;68:29-43.

4. Sun SY, Hail N Jr, Lotan R. Apoptosis as a novel target for cancer chemoprevention. J Natl Cancer Inst 2004;96:662-72.

5. Kinghorn AD, Farnsworth NR, Soejarto DD, Cordell GA, Swanson SM, Pezzuto JM. Novel strategies for the discovery of plant-derived anticancer agents. Pharmaceutical Biol 2003;41:53-67.

6. Kaufmann SH, Hengartner MO. Programmed cell death: Alive and well in the new millennium. Trends Cell Biol 2001;11:526-34.

7. Hengartner MO. The biochemistry of apoptosis. Nature 2000;407:770-6.
8. Rechinger KH. Flora Iranica, Vol. 72, Akademische Druckund Verlagsanstalt, Graz, Austria, 1987.

9. Mazloomifar H, Saber-Tehrani M, Rustaiyan A, Masoudi Sh. Constituents of the essential oil of echinophora platyloba DC growing wild in Iran. J Essent Oil Res 2004;16:284-5.

10. Mehta RG, Murillo G, Naithani R, Peng X. Cancer chemoprevention by natural products: How far have we come. Pharm Res 2010;27:950-61.

11. Shahneh FZ, Valiyari S, Azadmehr A, Hajiaghaee R, Yaripour S, Bandehagh A, et al. Inhibition of growth and induction of apoptosis in fibrosarcoma cell lines by Echinophora platyloba DC: In Vitro analysis. Adv Pharmacol Sci 2013;2013:512931.

12. Phelan MC. Basic techniques for mammalian cell tissue culture. Curr Protoc Cell Biol 1998;7:15-35.

13. Cole SP. Rapid chemosensitivity testing of human lung tumor cells using the MTT assay. Cancer Chemother Pharmacol 1986;17:259-63.

14. Shahneh FZ, Valiyari S, Baradaran B, Abdolalizadeh J, Bandehagh A, Azadmehr A, et al. Inhibitory and Cytotoxic activities of Salvia officinalis L. extract on human lymphoma and leukemia cells by induction of apoptosis. Adv Pharmaceut Bull 2013;3:51-5.

15. Frankfurt OS, Krishan A. Apoptosis enzyme-linked immunosorbent assay distinguishes anticancer drugs from toxic chemicals and predicts drug synergism. Chem Biol Interact 2003;145:89-99.

16. Sineh Sepehr K, Baradaran B, Mazandarani M, Khori V, Shahneh FZ. Studies on the cytotoxic activities of Punica granatum L. var. spinosa (apple punice) extract on prostate cell line by induction of apoptosis. ISRN Pharm 2012;2012:547942.

17. Shahneh FZ, Valiyari S, Azadmehr A, Hajiaghaee R, Baradaran B. Cytotoxic activities of Ferulago Angulata extract on human leukemia and lymphoma cells by induction of apoptosis. J Med Plants Res 2013;7:677-82.

18. Elmore S. Apoptosis: A review of programmed cell death. Toxicol Pathol 2007;35:495-516.

19. Fischer U, Schulze-Osthoff K. Apoptosis-based therapies and drug targets. Cell Death Differ 2005;12:942-61. 